\section{FLUID DYNAMICS}

\section{Flight of the dandelion}

Nature https://doi.org/10.1038/s41586-0180604-2 (2018)

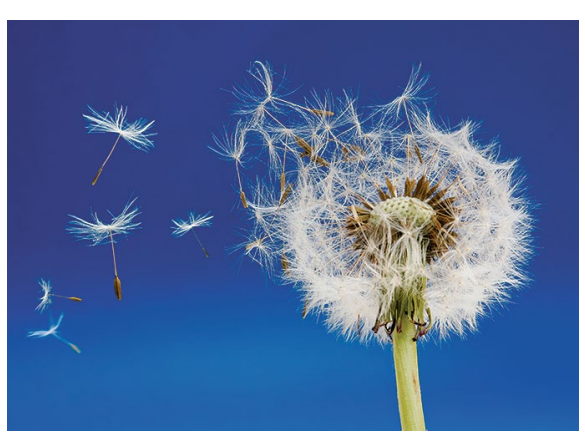

Credit: Brian Jackson/Alamy Stock Photo

Weed though it may be, the common dandelion is savvy when it comes to dispersing its seeds (pictured). Once separated from the head, the seeds remain airborne with the help of a bundle of dragenhancing bristles known as a pappus. This structure itself isn't an obvious bet for enabling the formidable distances the seeds cover: other species adopt wing-like structures in dispersal. But now, Cathal Cummins and colleagues have found that the bristly pappus induces a vortex of recirculating fluid that offers the requisite lift.

By visualizing the flow around the seeds, Cummins et al. found that for low Reynolds number, neighbouring bristles interact strongly due to the boundary layer around each filament. This in turn reduces airflow through the pappus, conferring a high drag coefficient. The team showed that a structure with a similar porosity could reproduce this flow behaviour, suggesting that pappus porosity is tuned to simultaneously stabilize the vortex and optimize aerodynamic loading. $A K$

https://doi.org/10.1038/s41567-018-0350-2

\section{ACADEMIC CAREERS}

\section{On the move}

J. R. Soc. Interface 15, 20180580 (2018)

Changing countries seems to be an unspoken rule for making a career in academia - but what is the actual impact of academic mobility? Alexander M. Petersen looked into this question by analysing the publication record of 26,170 physicists between 1980 and 2009.

Each physicist who relocated in that period of time - roughly $60 \%$ of the total - was paired with a non-mobile colleague who had a similar output prior to the move. On average, the comparison between these pairs showed that in the ten years after their move, researchers tend to accrue up to $17 \%$ more citations than their immobile counterparts. Additionally, the data revealed the crucial role of mobility in rewiring the global collaboration network, as moves lead to curtailing of old collaborations and the creation of new ones. This positive effect applies at all academic levels, and is not only to the benefit of the elite - making a case for better support of international travel for young researchers.

https://doi.org/10.1038/s41567-018-0351-1

\section{FREE-ELECTRON LASERS}

\section{The fast and the luminous} Nat. Commun. 9, 4025 (2018)

Serial femtosecond crystallography with ultra-intense X-ray pulses is one of the most promising analytical methods provided by large-scale free electron lasers. The

\title{
ASTROPARTICLE PHYSICS
}

\section{Greetings from the past Universe}

When the Universe was still in its infancy, nuclei such as helium or hydrogen isotopes began to form. Our current knowledge about this process - the so-called primordial nucleosynthesis - is limited, as the scattering off this particle 'soup' meant that the Universe was opaque to electromagnetic radiation. By comparing theoretical predictions with the measured abundance of the nuclei created, we can challenge our understanding of the early Universe. But are there no other ways to look back into the past?

Alexandre Ivanchik and Vlad Yurchenko think that we might be able to learn something from the contribution of neutron and tritium nuclear decays to the neutrino cosmic microwave background. They have predicted that these decays are the only source contributing to the spectrum for antineutrinos within a certain energy range. And as the neutrino cosmic microwave background was formed prior to the nucleosynthesis, an experimental observation of these antineutrinos would allow us to witness the creation of the first heavier elements.

microsecond interpulse separation afforded by the recently launched European X-ray Free-Electron Laser was anticipated to offer new opportunities by reducing the beamtime and sample volumes required - but its viability was not clear. Now, Anton Barty and colleagues have realized a setup capable of efficiently collecting high-quality diffraction patterns from small sample volumes.

Barty et al. exploited a custom-made version of a rapid full-frame detector and devised a novel high-speed jet to deliver and replenish the irradiated sample volume in the focal spot. They collected diffraction data from two different samples to benchmark their experiment at ångström resolution, demonstrating the ability to amass data from any member of the available megahertz X-ray pulse train. The unique method provides a key step toward future investigations of the temporal evolution of complex molecular structures.

https://doi.org/10.1038/s41567-018-0352-0

\section{QUANTUM METROLOGY}

\section{Be no exception}

Nat. Commun. https://doi.org/10.1038/s41467018-06477-7 (2018)

FL Dispersive measurement is the central idea behind modern sensor devices: the presence of a perturbation changes the original resonant frequency of the electromagnetic mode, which can be detected at very high precision. Recent investigations have suggested that the sensitivity could be further improved in a non-Hermitian system, making use of spectral degeneracies known as exceptional points. Now HoiKwan Lau and Aashish Clerk have identified the fundamental limit of this approach.

For a system to be non-Hermitian, coupling with an external reservoir is an essential ingredient. The dissipative dynamics is inevitably accompanied by noise, which was neglected in previous studies. By mapping the non-Hermitian sensing set-up to an open quantum system, Lau et al. were able to fully account for the noise effect, and derive the limit that constrains sensing protocols based on non-Hermitian Hamiltonians. Within their model, the improvement arising from exceptional points can be achieved by simply adding gain to conventional set-up without any such points in the system.

https://doi.org/10.1038/s41567-018-0354-y 